A. Tammaro ${ }^{1}$, M. Muscianese ${ }^{1}$, C. Chello $^{1}$, F.R. Parisella ${ }^{2}$, S. Persechino ${ }^{1}$, C. Goebel ${ }^{3}$, A.A. Gaspari ${ }^{4}$

\title{
Cross-elicitation responses to 2-methoxymethyl- p-phenylenediamine in p-phenylenediamine highly allergic volunteers using allergy alert test: the Italian experience
}

\author{
${ }^{1}$ Department of Dermatology, University of Rome Sapienza, Sant'Andrea Hospital, Rome, Italy \\ ${ }^{2}$ University of Queensland, Brisbane, Australia \\ ${ }^{3}$ Toxicology Department, Coty Professional Beauty, Darmstadt, Germany \\ ${ }^{4}$ Department of Dermatology and Cutaneous Biology, Sidney Kimmel Medical College, Thomas Jefferson University, \\ Philadelphia, PA, USA
}

\section{KEYWORDS}

p-phenylenediamine; allergic reaction; 2-methoxymethyl-pphenylenediamine

\author{
Corresponding author \\ Antonella Tammaro \\ Sant'Andrea Hospital \\ Via di Grottarossa 1035, 00189 \\ Roma, Italy \\ Phone: +39 0633775269 \\ E-mail: tammaroantonella@gmail.com
}

\section{Doi}

10.23822/EurAnnACI.1764-1489.82

\begin{abstract}
Summary
Background. Allergic contact dermatitis after exposure to p-phenylenediamine (PPD)-containing hair dye products is a common and important clinical problem. Because there is a high rate of cross-elicitation of allergic contact dermatitis to other important hair dye products (such as p-toluene diamine [PTD] and other aminophenol hair dyes) in PPD allergic patients, safer alternative dyes with excellent hair coloring options are needed. We studied 2-methoxy meth$y l-P P D(M e-P P D)$, a chemical derivative of PPD for tolerance versus cross-elicitation in a cohort of eight PPD-allergic volunteers. Objective. To study tolerance to Me-PPD in a PPD highly allergic Italian cohort. Methods. Eight volunteers with a history of contact dermatitis to hair dyes or other PPD-containing chemicals and positive patch tests to 1\% PPD in petrolatum, were recruited to study their immediate and delayed skin reactivity to PPD, vehicle control and 2-methoxy-methyl-PPD (Me-PPD), using the allergy alert test (simulating hair dyeing conditions) on volar forearm skin. This is a short-contact open patch test. Results. All eight volunteers reacted to PPD allergy alert test (100\%); none reacted to vehicle (0\%), and seven of eight reacted to $\mathrm{Me}$ PPD allergy alert test (88\%). However, in those seven volunteers who exhibited cross-elicitation to $M e-P P D$, their aggregate skin test reactivity to Me-PPD was significantly less than that of PPD (figure 3, $p<0.0062$, highly significant, paired two-tailed, students t test). Conclusions. MePPD may offer a safer alternative for PPD-allergic patients with an absent or reduced elicitation response in the allergy alert test simulating hair dye use conditions. Even patients with strong patch test reactions, with appropriate selection by allergy alert test and counselling, may be able to tolerate hair dyeing with Me-PPD containing products.
\end{abstract}

\section{Introduction}

Para-phenylenediamine (PPD) is a component of permanent hair dyes, and may be used to dye textiles and fur. Allergic contact dermatitis to PPD has increased significantly in the general population over the last ten years. The prevalence of positive patch-test results to PPD has been in the range of $4 \%$ to $5 \%$ in a large series of dermatitis patients (1-4).

PPD and the structurally related compound p-toluene-diamine (PTD) are considered the most important allergens associated with allergic contact dermatitis related to the use of hair dyes. There is a positive relationship between an elicitation response 
to PPD and concomitant reactions to other chemically related components of oxidative hair colors, such as para-substituted benzene. This phenomenon has been termed "cross-elicitation", whereby sensitization to PPD or PTD elicits patch test reactivity to the other compound, even though there has not been a prior exposure. This phenomenon occurs because of the chemical similarities between the two compounds.

Clinically, there may be a severe acute dermatitis involving the face, eyelids and neck with only minimal scalp involvement in PPD allergic individuals that use PPD-containing hair dyes. Recently, a PPD derivative called 2-methoxymethyl-p-phenylenediamine (Me-PPD) has been developed by the introduction of a methoxymethyl side chain into PPD parent molecule. This molecule is a hair dye precursor with excellent hair coloring performance, and exhibits significantly reduced skin sensitizing properties compared to PPD or PTD. Therefore, Me-PPD has favorable properties, that include reduced propensity to allergy induction with excellent color results when used as a hair dye (5). Herein, we investigated eight PPD-allergic patients to evaluate the risk of develop cross-elicitation responses to Me-PPD under conditions that simulate the hair-dyeing process. We selected a cohort of patients with strong PPD allergy (by clinical symptoms, and with confirmation by strong patch test reactivity (PPD 2-3+ reactivity).

\section{Materials and methods}

All volunteers were recruited from the Department of Dermatology at the University of Rome Sapienza, Sant'Andrea Hospital. Eight female volunteers aged between 32 and 47, with a documented history of signs and symptoms consistent with PPD allergy were enrolled. All the patients exhibited a positive standard patch test result to PPD (1\% PPD in petrolatum [100 $\mu \mathrm{l}])$. Patch testing and allergy alert testing was scored, according to the International Contact Dermatitis Research Group (ICDRG) classification system, between $2+$ and $3+$ (6 patients were scored as $2+, 4$ patients were scored as $3+$ ). There were no volunteers that exhibited $1+$ reactivity or weaker. All of the volunteers had experienced allergic contact dermatitis related to the use of hair dyes in the past. Table I summarizes the eight volunteers enrolled in our study.

We excluded PPD-allergic patients with a history suggestive of severe allergic reactions, such as anaphylaxis or contact urticaria. Volunteers were not permitted to use topical corticosteroids or oral antihistamines one month prior to Visit 1, or systemic corticosteroids or immunosuppressive agents three months prior to Visit 1. Other exclusion criteria were the history of drug or alcohol abuse within the past 6 months, as determined by the medical record or patient interview. Any scarring, infection, or skin disease in the area being patch tested (ventral forearms) were also exclusion criteria. Other exclusion criteria included an inability to make study visits or anticipated poor compliance, pregnant females or nursing mothers, any history or evidence of severe illness or any other condition that would render the volunteer unsuitable for the study.

Eligible women of reproductive age were required to have a negative urine pregnancy test at screening. Eight volunteers enrolled. The following compounds were used in this study:

i) PPD (1\% PPD in petrolatum under a FINN chamber, the standard diagnostic patch test). A positive patch test to PPD (along with a history of intolerance to PPD-containing hair dye) was the major criterion for enrollment in this study;

ii) Me-PPD hair dye tint: vehicle (Koleston Perfect formula without fragrance), hair dye precursor (4\% Me-PPD, free base), couplers (3.6\% 2-methylresorcinol and 1.9\% 2-methyl-5-hydroxyethylaminophenol). This chemical derivative of PPD was the unknown in this study, which was evaluated for cross-elicitation or tolerance in volunteers with known PPD allergy related to prior use of hair dyes containing PPD;

iii) PPD hair dye tint: vehicle (Koleston Perfect formula without fragrance), hair dye precursor (4\% PPD, free base), cou-

Table I - Summary of characteristics of volunteers enrolled in study.

\begin{tabular}{|c|c|c|c|c|c|}
\hline Patient no. & Skin type & Ethnicity & Age/gender & PPD patch test & Route of exposure \\
\hline 1 & II & caucasian & 31/female & $3+$ & hair dye \\
\hline 2 & II & caucasian & 51/female & $3+$ & hair dresser (hair dye) \\
\hline 3 & II & caucasian & 39/female & $3+$ & hair dye \\
\hline 4 & II & caucasian & 60/female & $3+$ & hair dye \\
\hline 5 & II & caucasian & 33/female & $3+$ & hair dye \\
\hline 6 & II & caucasian & 35/female & $2+$ & street tattoo \\
\hline 7 & II & caucasian & 32/female & $2+$ & cosmetics \\
\hline 8 & II & caucasian & 34/female & $3+$ & hair dye \\
\hline
\end{tabular}


plers (3.6\% 2-methylresorcinol and 1.9\% 2-methyl-5-hydroxyethylaminophenol), PPD served as a positive control for the allergy alert test;

iv) vehicle control (Koleston Perfect formula without fragrance). The vehicle (containing no PPD) served as a negative control, to prove that PPD was responsible for the positive allergy alert test (in addition to the standard patch test described above).

Immediately prior application to the volunteers' forearms, the hair dye tints (either PPD or Me-PPD) and the vehicle control were mixed with an equal volume of hydrogen peroxide solution $(6 \%[\mathrm{w} / \mathrm{w}]$ Welloxon) at a $1: 1$ ratio with the hair dye tints, resulting in a final concentration of 2\% Me-PPD and PPD in the allergy alert test, respectively. This is the exact methodology used in a prior study (8).

The PPD ( $1 \%$ in petrolatum) patch test was used under a FINN chamber occlusion, with removal of the patch test after 48 hours and readings performed at 48 and 72 hours. This standard patch test had been performed prior to enrollment in this study.

Me-PPD and PPD (formulations with couplers and hydrogen peroxide solution) and vehicle alone were tested using a modified protocol: after 30 minutes following placement of a $1 \mathrm{~cm}^{2}$ area of an open application to the ventral forearm, the Me-PPD, PPD, and vehicle formulations were gently rinsed off with a hypoallergenic soap and water. This simulates hair dyeing conditions (allergy alert test).

Skin evaluation readings for reactivity to PPD, Me-PPD and vehicle control (allergy alert test) were performed at 30 minutes, 48 hours and 72 hours.

The International Contact Dermatitis Research Group (ICDRG) scoring system was used to grade patch testing results (6). Scores range from 0 to $3+: 0(-)=$ negative reaction; ?+ = doubtful reaction / erythema only; $1+(+)=$ weak (non-vesicular) positive allergic reaction (erythema, infiltration, and possible papules); $2+(++)=$ strong $($ vesicular) positive reaction (erythema, infiltration, papules, and vesicles); $3+(+++)=$ extreme positive allergic reaction; bullous reaction.

\section{Statistical analysis}

To compare the allergy alert test results of the eight volunteers against PPD and Me-PPD, we used the Graph Pad Prism Software Program (Graph Pad Software, Inc., La Jolla, CA.). Because we compared PPD and Me-PPD reactivity within each of the eight volunteers, we used the paired, two-tailed student's t-test. P values $<0.05$ were considered significant.

\section{Results}

We evaluated the potential of Me-PPD to cross-elicit allergic contact dermatitis on the ventral forearm skin of eight female volunteers with patch-test proven PPD-allergy and a history of clinical hair dye intolerance (a standard definition of PPD allergy related to the use of PPD containing hair dye). To assess the immediate reactivity of the skin, we performed readings 30 minutes after the removal of Me-PPD, PPD (formulations with couplers and hydrogen peroxide solution) and vehicle. At the 30-minute reading, none of the eight volunteers exhibited a positive skin test. This was interpreted as negative immediate skin reactivity to PPD, Me-PPD and vehicle (data not shown).

Table II summarizes the delayed reactivity to PPD, vehicle and Me-PPD on allergy alert test in the context of their diagnostic PPD patch test. All eight volunteers reacted to PPD allergy alert test (100\%); none reacted to vehicle $(0 \%)$, and seven of eight reacted to Me-PPD allergy alert test (88\%). Figure 1 summarizes the cross-elicitation of the eight volunteers using the allergy alert test for Me-PPD at the final $(72$ hour) read. Of the six volunteers with $3+$ (extreme) reactivity to PPD standard patch tests, five exhibited cross-elicitation to Me-PPD on allergy alert. Of the two volunteers with $2+$ (strong) reactivity to PPD standard patch tests, both (100\%) exhibited cross-elicitation to Me-PPD.

Figure 2 summarizes tolerance (non-reactivity) to Me-PPD in our eight allergic-PPD volunteers. Of the two volunteers with $2+$ PPD reactivity on standard patch testing, none $(0 / 2,0 \%)$ was tolerant. Of the six volunteers with $3+$ PPD reactivity on standard patch testing, one $(1 / 6,16 \%)$ was tolerant.

We compared the strength of the reactivity of Me-PPD to PPD in those seven volunteers who reacted to both compounds. In five of seven volunteers, the reaction to PPD allergy alert test was stronger than that of Me-PPD (not shown). In two of seven volunteers, the reaction to PPD allergy alert test was the same as Me-PPD (not shown). None of the seven volunteers exhibited a stronger allergy alert test to Me-PPD compared to

Table II - Summary of allergy alert test for PPD, Vehicle and MePPD (number positivelnumber tested).

\begin{tabular}{cccc}
\hline $\begin{array}{c}\text { Strength of PPD } \\
\text { patch test }\end{array}$ & $\begin{array}{c}\text { PPD } \\
\text { allergy } \\
\text { alert }\end{array}$ & $\begin{array}{c}\text { Vehicle } \\
\text { allergy alert }\end{array}$ & $\begin{array}{c}\text { Me-PPD } \\
\text { allergy alert }\end{array}$ \\
\hline++ & $6 / 6^{1}$ & $0 / 6$ & $5 / 6$ \\
\hline++ & $2 / 2$ & $0 / 2$ & $2 / 2$ \\
\hline+ & $\mathrm{n} / \mathrm{a}^{2}$ & $\mathrm{n} / \mathrm{a}$ & $\mathrm{n} / \mathrm{a}$ \\
\hline$\%$ positive & $100 \%$ & $0 \%$ & $88 \%$
\end{tabular}

${ }^{1}$ Number of positive on allergy alert test ( $1+$ to $3+/$ number tested);

${ }^{2} \mathrm{n} / \mathrm{a}=$ not applicable (there were no volunteers with moderate reactivity to PPD on patch testing). 
PPD (not shown). In aggregate, the mean reactivity to PPD was stronger than Me-PPD on allergy alert test (figure 3a). This difference was highly significant, despite the small sample size ( $p<0.0062$, two tailed, paired $t$-test with eight paired val-

Figure 1. Final (72 h reading) scores of Me-PPD allergy alert test in the context of PPD patch test results in eight volunteers enrolled in the present study. Volunteers are grouped by their diagnostic patch test to $1 \%$ PPD, either $2+$ or $3+$ (there were no volunteers who exhibited $1+$ reactivity).

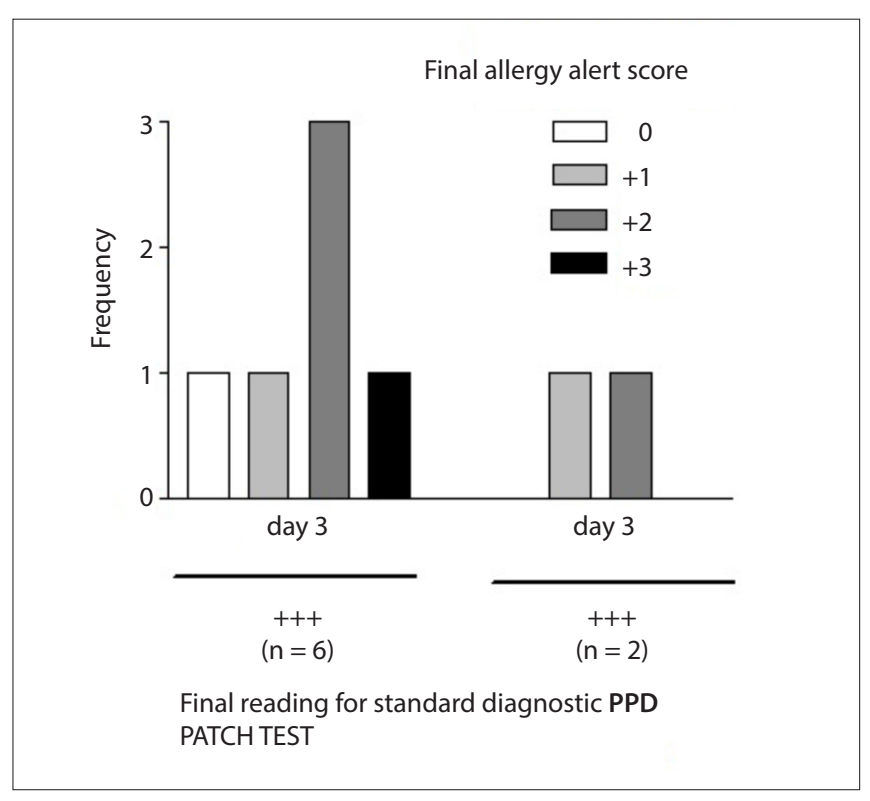

ues). The results of the 72-hour reading of allergy alert test in one of the volunteers enrolled in this study are depicted. This includes PPD, vehicle and Me-PDD, see figure $\mathbf{3 b}$, revealing a stronger reaction to PPD $(2+)$ than to Me-PPD (1+).

Figure 2. Tolerance to Me-PPD allergy alert test in context of PPD patch test results. Of the eight volunteers in this study, two exhibited moderate (2+) patch test reactivity to $1 \%$ PPD diagnostic patch test, and none of these two subjects were tolerant to Me-PPD (O\%). The remaining six subjects exhibited extreme (3+) reactivity to $1 \%$ diagnostic patch test, and one of six (16\%) were tolerant of $\mathrm{Me}$ $P P D$. The overall rate of tolerance in this cohort of highly reactive PPD volunteers was $12 \%$ (one of eight).

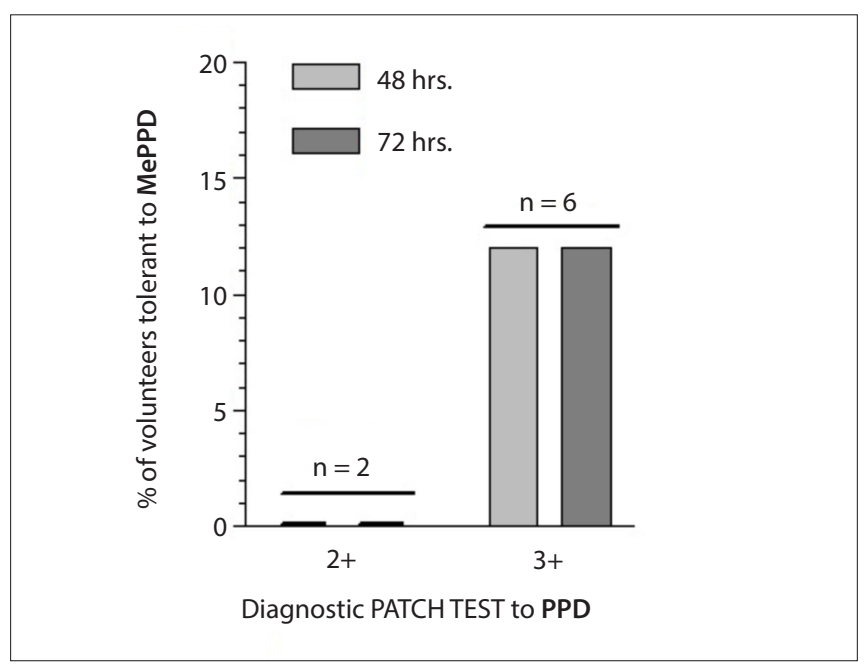

Figure 3. Comparison of PPD and Me-PPD allergy alert test results in eight volunteers. $a$ - the aggregate (mean +/- SD) allergy alert scores for $P P D$ and Me-PPD were compared. The mean reactivity of PPD was stronger than that of Me-PPD. The reactivity to Me-PPD was signifcantly less than that of PPD (highly significant, $p<0.0062$, two tailed, paired $t$-test, with eight pairs); $\boldsymbol{b}$ - allergy alert test at 72 hours in volunteer who was not tolerant of Me-PPD. Her reaction to PPD was stronger than that of Me-PPD. There was no reaction to the vehicle control.
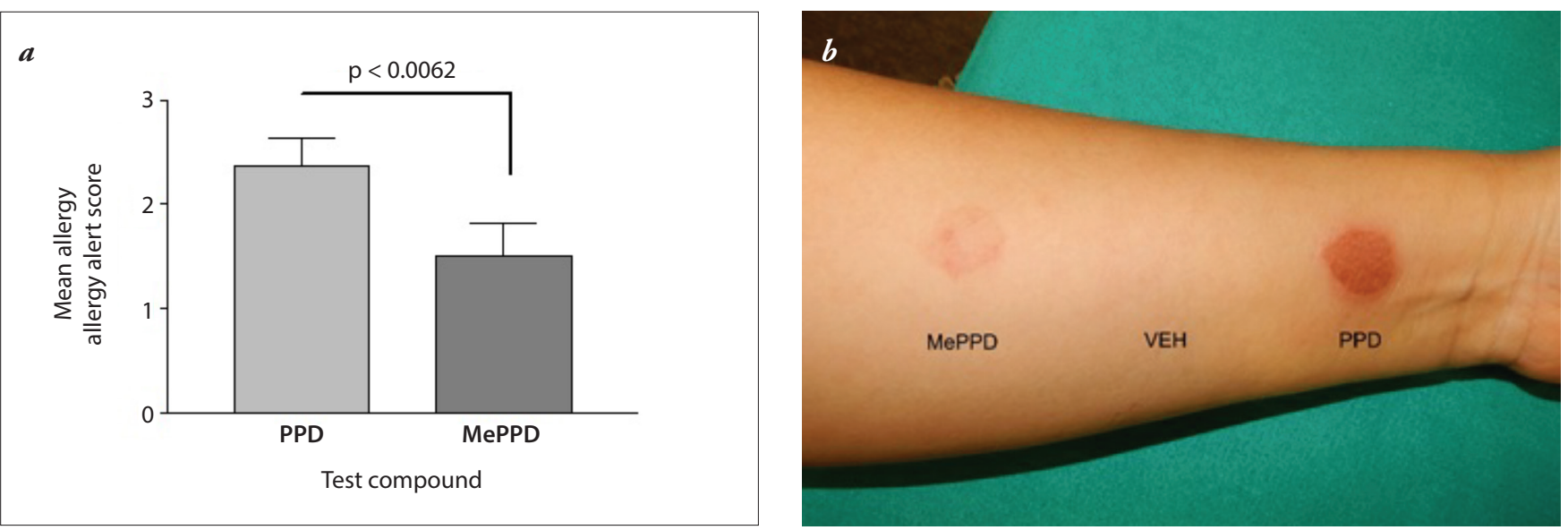


\section{Discussion}

PPD is widely used as a permanent hair dye, but it may also been found in textile or fur dyes, temporary tattoos, photographic developer and lithography plates, photocopying and printing inks, black rubber, oils, greases and gasoline. Despite its presence in a variety of products, exposure to PPD-containing hair dyes is the main cause of PPD allergy (7).

The introduction of a methoxymethyl side chain into PPD created a hair dye precursor with excellent hair coloring performance. The Me-PPD has been studied in pre-clinical predictive assays to determine its relative sensitization capacity compared to the parent compound, PPD. Using the local lymph node assay (a standard in vivo mouse sensitization assay), the effective concentration of Me-PPD necessary to induce an immune response 3-fold above vehicle control (EC3 value) in the local lymph node assay (LLNA) was $4.3 \%$, indicating a moderate skin sensitizing potency compared to values of $0.1 \%$ and $0.17 \%$ for PPD and PTD. Both PPD and PTD are considered to be strong sensitizers, and thus induce a response in the local lymph node assay at much lower concentrations compared to Me-PPD. These pre-clinical data indicate that Me-PPD has significantly reduced skin sensitizing properties compared to PPD or PTD (8).

Blomeke et al. (9) studied the sensitivity of the allergy alert test in a cohort of patch test-proven PPD allergic volunteers. She demonstrated that under stimulated hair coloring conditions (that is, the skin allergy alert test, a short contact open patch test, see methods), the rate of cross-elicitation to Me-PPD (30\%) was lower than to PPD (84\%). On this basis, we have conducted a study to investigate if patients with a documented history of allergic contact dermatitis to PPD assessed by positive patch test results, develop a cross-elicitation response to Me-PPD under conditions mimicking hair dyeing. We enrolled eight female patients who had experienced hair allergic contact dermatitis to PPD-containing hair dye (confirmed by standard diagnostic patch testing).

In past studies, tolerance to cross-elicitation to Me-PPD by allergy alert testing in PPD allergic volunteers was inversely proportional to PPD patch test reactivity (9). That is, tolerance to Me-PPD under hair-dyeing conditions was between 50-100\% in volunteers with $1+$ PPD patch test reactivity; $40-85 \%$ in volunteers with $2+$ PPD patch test reactivity, and $33-50 \%$ in volunteers with $3+$ PPD reactivity $(5,8-10)$. In the present study, we enrolled only volunteers with a positive standard patch test to PPD scored between +2 and +3 , according to the International Conctact Dermatitis Research Group (ICDRG) classification system. The overall rate of tolerance to Me-PPD in our highly PPD-reactive cohort was $12 \%$. The lower rate of tolerance to Me-PPD is due to the small population size, that was comprised only of strong and very strong PPD reactors. The previous three studies had significant numbers $(53 \%, 35 \%$ and $9 \%)$ of volunteers with moderate reactivity to PPD on patch testing. In our study, there were no moderate PPD reactors $(1+)$, only strong $(2+)$ and extreme reactors $(3+)$.

Interestingly, our only Me-PPD tolerant volunteer was an extreme PPD reactor (3+ on PPD patch testing), which also occurred in previous studies (33 to $50 \%$ of volunteers with extreme patch test $[3+]$ results to $\mathrm{PPD})$. Since allergic contact dermatitis to PPD is T-lymphocyte mediated (11-14), it is likely that the mechanism for the cutaneous tolerance involves T-lymphocyte tolerance. The immunology of tolerance to Me-PPD in the PPD highly allergic individual warrants further study, as it may be an example of powerful suppressor mechanisms. Most likely, this involves suppressor mechanism(s) such as T-regulatory cells $(15,16)$, which control potent T-effector/T-memory mechanisms in such highly PPD-allergic individuals.

Lastly, in those PPD-allergic volunteers who were not tolerant of Me-PPD, their relative reactivity to Me-PPD on allergy alert testing was, in most cases, less than PPD on allergy alert testing (figure 3a). These data are in line with the results from a recent study from the USA (8).

These data confirm that it may be possible to safely use MePPD even in those individuals who are highly allergic to PPD (i.e., complete tolerance to Me-PPD, as demonstrated by one of eight volunteers in our study). This may only be possible with careful patient selection with allergy alert test and counseling being key components to assure consumer safety. Those subjects who exhibit any reactivity to Me-PPD on allergy alert test (even if this reactivity is less than that of PPD on allergy alert test) should not use a Me-PPD containing hair dye.

Limitations of this study are the small sample size $(\mathrm{n}=8)$. Further long-term studies are needed, as this study was only a single exposure.

\section{Acknowledgements}

The authors thank Dr. Richard M.C. Sutton (Procter and Gamble) for providing the materials utilized for the allergy alert test.

\section{Statement of ethics}

Subjects (or their parents or guardians) have given their written informed consent. The study protocol has been approved by the research institute's committee on human research.

\section{Conflict of Interest}

Dr. Carsten Goebel is an employee of Coty, Inc.

\section{Funding Sources}

The authors received no specific funding for this work. 


\section{References}

1. Fregert S, Hjorth N, Magnusson B, Bandmann HJ, Calnan CD, Cronin E, Malten K, Meneghini CL, Pirilä V, Wilkinson DS. Epidemiology of contact dermatitis. Trans St Johns Hosp Dermatol Soc $1969 ; 55(1): 17-35$.

2. Patel S1, Basketter DA, Jefferies D, White IR, Rycroft RJ, McFadden JP, Ho SY. Patch test frequency to p-phenylenediamine: follow up over the last 6 years. Contact Dermatitis 2007; 56(1):35-37.

3. Diepgen TL, Naldi L, Bruze M, Cazzaniga S, Schuttelaar M-L, Elsner P, Goncalo M, Robert Ofenloch R and Svensson A. Prevalence of Contact Allergy to p-Phenylenediamine in the European General Population. Journal of Investigative Dermatology 2016; 136:409-415.

4. Krasteva M, Bons B, Ryan C, and Gerberick GF. Consumer Allergy to Oxidative Hair Coloring Products: Epidemiologic Data in the Literature. Dermatitis 2009; 20(3):123-141.

5. Goebel C, Troutman J, Hennen J, Rothe H, Schlatter H, Gerberick GF, Blömeke B.Introduction of a methoxymethyl side chain into p-phenylenediamine attenuates its sensitizing potency and reduces the risk of allergy induction. Toxicol Appl Pharmacol 2014; 274(3):480-487.

6. Fregert S. Manual of Contact Dermatitis. On behalf of the International Contact Dermatitis Research Group and the North American Contact Dermatitis Group. Copenhagen: Munksgaard Publishers, 1981.

7. Schnuch A1, Lessmann H, Frosch PJ, Uter W. para-Phenylenediamine: the profile of an important allergen. Results of the IVDK. Br J Dermatol 2008; 159(2):379-386.
8. Zahir A, Kindred C, Blömeke B, Goebel C, Gaspari AA.Tolerance to a Hair Dye Product Containing 2-Methoxymethyl-P-Phenylenediamine in an Ethnically Diverse Population of P-Phenylenediamine-Allergic Individuals. Dermatitis 2016; 27(6):355-361.

9. Blömeke B, Pot LM, Coenraads PJ, Hennen J, Kock M, Goebel C. Cross-elicitation responses to 2-methoxymethyl-p-phenylenediamine under hair dye use conditions in p-phenylenediamine-allergic individuals. Br J Dermatol 2015; 172(4):976980.

10. Goebel C1, Coenraads PJ, Rothe H, Kunze G, Kock M, Schlatter H, Gerberick GF, Blömeke B. Elicitation of the immune response to p-phenylenediamine in allergic patients: the role of dose and exposure time. Br J Dermatol 2010; 163(6):12051211.

11. Voisin GA. Immunity and tolerance: a unified concept. Cell Immunol 1971; 2(6):670-689.

12. Polak L, Frey JR. Tolerance and desensitization in experimental eczema. Curr Probl Dermatol 1972; 4:146-177.

13. Appleman LJ, Boussiotis VA. T cell anergy and costimulation. Immunol Rev 2003; 192:161-180.

14. Klein J. What causes immunological nonresponsiveness? Immunol Rev 1984; 81:177-202.

15. Cavani A. Immune regulatory mechanisms in allergic contact dermatitis and contact sensitization. Chem Immunol Allergy 2008; 94:93-100.

16. Gober MD, Gaspari AA. Allergic contact dermatitis. Curr Dir Autoimmun 2008; 10:1-26. 